\title{
CORRECTION
}

\section{Correction: Transdifferentiation of tumor infiltrating innate lymphoid cells during progression of colorectal cancer}

Shuo Wang, Yuan Qu, Pengyan Xia, Yi Chen, Xiaoxiao Zhu, Jing Zhang, Guan Wang, Yong Tian, Jianming Ying and Zusen Fan

Cell Research (2020) 30:630; https://doi.org/10.1038/s41422-020-0352-3

Correction to: Cell Research https://doi.org/10.1038/s41422-0200312-y, published online 04 May 2020

We apologize for the mistake of the author information. Dr. Zusen Fan should be one of the correspondence authors, and Dr. Yong Tian is one of the co-first authors. The correct information is as follows. This correction does not affect the description of the results or the conclusion of this work.

Correspondence: Shuo Wang (wangshuo@im.ac.cn) or Jianming Ying (jmying@cicams.ac.cn) or Zusen Fan (fanz@moon.ibp.ac.cn) These authors contributed equally: Shuo Wang, Yuan Qu, Pengyan Xia, Yi Chen, Yong Tian. 